Mathematical Modelling and Analysis

Volume 21 Number 3, May 2016, 283-303

http://dx.doi.org/10.3846/13926292.2016.1152611

(c) Vilnius Gediminas Technical University, 2016
Publisher: Taylor\&Francis and VGTU

http://www.tandfonline.com/TMMA

ISSN: $1392-6292$

eISSN: $1648-3510$

\title{
Properties of a Composite Material with Mixed Imperfect Contact Conditions
}

\section{Luís Filipe Pinheiro de Castro $^{a}$ and Ekaterina Pesetskaya ${ }^{b}$}

${ }^{a}$ CIDMA and Department of Mathematics, University of Aveiro

Aveiro, Portugal

${ }^{b}$ Andrea Razmadze Mathematical Institute, Tbilisi State University

Tbilisi, Georgia

E-mail(corresp.): kate.pesetskaya@gmail.com

E-mail: castro@ua.pt

Received October 11, 2015; revised February 04, 2016; published online May 15, 2016

\begin{abstract}
We present an analytical solution of a mixed boundary value problem for an unbounded 2D doubly periodic domain which is a model of a composite material with mixed imperfect interface conditions. We find the effective conductivity of the composite material with mixed imperfect interface conditions, and also give numerical analysis of several of their properties such as temperature and flux.
\end{abstract}

Keywords: unbounded 2D doubly periodic composite material, functional equations, effective conductivity, non-ideal contact condition.

AMS Subject Classification: 30E25; 30D05; 31A05; 35B27; 39B32; 74E30; 74G10; 74M15; 74Q05; 74S70.

\section{Introduction}

The use of composite materials is growing very fast. In part, this is due to the circumstance of being possible to choose their specific properties so that they exactly meet the requirements of a particular application. In view of this, detailed knowledge about different types of properties of composite materials (upon some of their characteristics) has a key role in the ongoing research being conducted in this field.

The present work is concerned with an analytical analysis of a 2D composite material model. The theory and technique for such analysis of composite material properties are under development. Recently, in [16], it was derived an explicit formula allowing to compute the sensitivity of large scale conductivity of a composite material to parameters describing its microstructure (such as material microstructure properties or a set of data describing the geometrical shape of inclusions). By means of the asymptotic homogenization method, analytical formulae were obtained in [11] for the effective thermoelastic coefficients 
of a fiber-reinforced periodic elastic composite with hexagonal cell, where the constituents exhibit transverse isotropic properties. On the base of the twoscale asymptotic homogenization method, the effect of the interface properties on the effective conductivity and temperature/flux field of a granular composite with a simple cubic array of spherical inclusions was analytically studied in [2].

According to homogenization theory (cf. $[1,12]$ ), fibrous composite materials with a large number of cylindrical unidirectional inclusions can be geometrically represented as a $2 \mathrm{D}$ doubly periodic model with disjoint inclusions, and their properties can be studied on the basis of a representative cell containing all material features. From the mathematical point of view, a 2D model with disjoint inclusions is a multiply connected domain. In case of steady-state conduction, the problem of determination of material properties is equivalent to the problem of finding a potential function satisfying the Laplace equation in each inner point of the model and satisfying certain conditions on the boundary of a considered domain. Ideal, imperfect or other contact conditions can be considered as boundary conditions. In several different situations, the obtained mixed boundary value problem can be equivalently reduced to a conjugation problem for analytical functions. To solve this conjugation problem, the method of functional equations, developed in [14], has proven to be a very powerful technique.

Indeed, the method of functional equations was earlier used in some papers devoted to analytical solution of mixed boundary value problems for the Laplace equation. Mixed boundary value problems with ideal contact conditions have been investigated e.g. in $[4,13,15,17,18]$. In $[6,10]$, mixed boundary value problems with soft imperfect contact conditions were analytically solved using also a consequent adaptation of this method. Moreover, in the recent paper [5], the authors modified the method of functional equations in the case of stiff contact conditions.

The novelty of the present work is centred on the fact that we are considering even more involved stiff imperfect conditions by admitting mixed (soft and stiff) imperfect contact conditions. In this case, we find in explicit form material characteristics such as temperature, flux and the effective conductivity for a $2 \mathrm{D}$ composite material model with circular disjoint inclusions. The obtained formula contains all parameters of the considered model such as the conductivity, radii, centers of the inclusions, the conductivity of the matrix, and also special Eisenstein functions.

The structure of the obtained formulae allows us to implement a detailed numerical analysis of those properties, by using the Maple 14 software. Here, as of particular significance, we emphasize the obtained detailed description of the associated temperature and flux distributions.

\section{Formulation of the problem}

We consider a lattice in the complex plane $\mathbb{C} \cong \mathbb{R}^{2}$ of a complex variable $z=x+\imath y$. The representative cell is the unit square

$$
Q_{(0,0)}:=\left\{z=t_{1}+\imath t_{2} \in \mathbb{C}:-\frac{1}{2}<t_{p}<\frac{1}{2}, p=1,2\right\} .
$$


Let $\mathcal{E}:=\bigcup_{m_{1}, m_{2}}\left\{m_{1}+\imath m_{2}\right\}$ be the set of the lattice points, where $m_{1}, m_{2} \in \mathbb{Z}$. The cells corresponding to the points of the lattice $\mathcal{E}$ will be denoted by

$$
Q_{\left(m_{1}, m_{2}\right)}=Q_{(0,0)}+m_{1}+\imath m_{2}:=\left\{z \in \mathbb{C}: z-m_{1}-\imath m_{2} \in Q_{(0,0)}\right\} .
$$

It is considered the situation when mutually disjoint disks (inclusions) of different radii $D_{k}:=\left\{z \in \mathbb{C}:\left|z-a_{k}\right|<r_{k}\right\}$ with the boundaries $\partial D_{k}:=\{z \in \mathbb{C}$ : $\left.\left|z-a_{k}\right|=r_{k}\right\}(k=1,2, \ldots, N)$ are located inside the cell $Q_{(0,0)}$ and periodically repeated in all cells $Q_{\left(m_{1}, m_{2}\right)}$. Let us denote by

$$
D_{0}:=Q_{(0,0)} \backslash\left(\bigcup_{k=1}^{N} D_{k} \cup \partial D_{k}\right)
$$

the connected domain obtained by removing of the inclusions from the cell $Q_{(0,0)}$ (see Fig. 1).

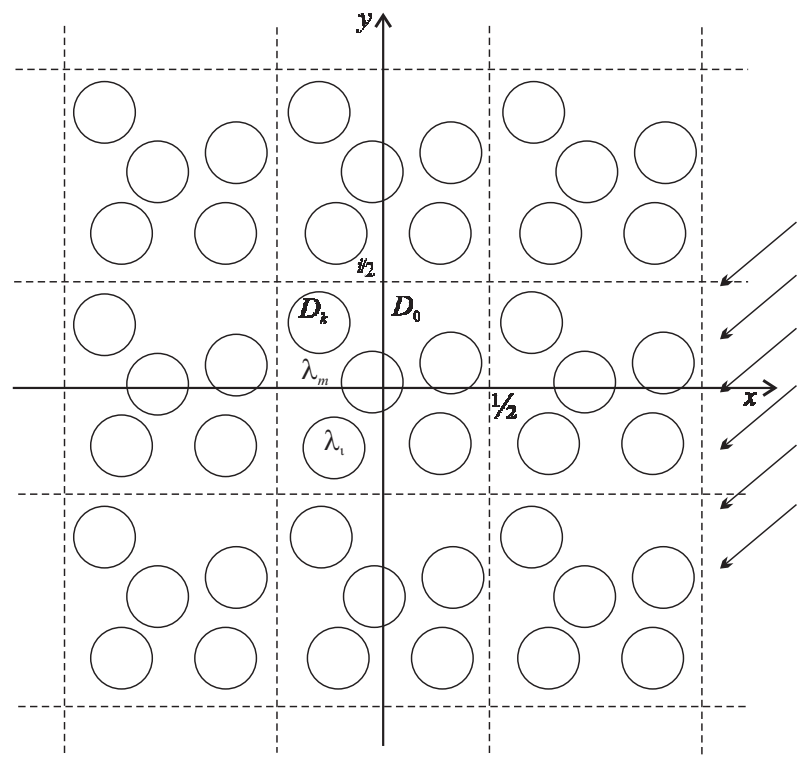

Figure 1. The representative cell $Q_{(0,0)}$ within doubly periodic composite.

Let us consider the problem of determination of the effective conductivity of an unbounded doubly periodic composite material with matrix

$$
D_{\text {matrix }}=\bigcup_{m_{1}, m_{2}}\left(\left(D_{0} \cup \partial Q_{(0,0)}\right)+m_{1}+\imath m_{2}\right)
$$

and inclusions

$$
D_{i n c}=\bigcup_{m_{1}, m_{2}} \bigcup_{k=1}^{N}\left(D_{k}+m_{1}+\imath m_{2}\right)
$$


occupied by materials of conductivities $\lambda_{m}>0$ and $\lambda_{k}>0$, respectively.

This problem is equivalent to the determination of the potential of the corresponding fields, i.e., a function $T$ satisfying the Laplace equation in each component of the composite material,

$$
\Delta T(z)=0, \quad z \in D_{\text {matrix }} \cup D_{\text {inc }},
$$

which have to satisfy the following boundary conditions on all $\partial D_{k}, k=$ $1,2, \ldots, N$ :

$$
\begin{gathered}
\gamma_{k}\left(T_{m}(t)-T_{k}(t)\right)=\lambda_{k} \frac{\partial T_{k}}{\partial n}(t) \\
\lambda_{m} \frac{\partial T_{m}}{\partial n}(t)-\lambda_{k} \frac{\partial T_{k}}{\partial n}(t)=\beta_{k} \frac{\partial^{2} T_{k}}{\partial s^{2}}(t), \quad t \in \bigcup_{m_{1}, m_{2}} \partial D_{k},
\end{gathered}
$$

where $\gamma_{k}>0, \beta_{k}<0$ are given parameters (the so-called resistent coefficients), the vector $n=\left(n_{1}, n_{2}\right)$ is the outward unit normal vector to $\partial D_{k}$, the vector $s$ is the outward unit tangent vector to $\partial D_{k}$, and

$$
T_{m}(t):=\lim _{z \rightarrow t, z \in D_{0}} T(z), \quad T_{k}(t):=\lim _{z \rightarrow t, z \in D_{k}} T(z) .
$$

The conditions (2.2)-(2.3) form the mixed imperfect contact conditions.

Let us mention that the usually adopted ideal contact conditions consist in demanding the continuity of the temperature and the thermal flux. Here, we use a relaxation of these conditions and allow certain discontinuities. Namely, in (2.2), according to the Fourier's law, we assume that the temperature jump across the boundary is proportional to the thermal flux of an inclusion. The second condition (2.3) comes from the influence of some "hidden" small thickness interface layer between the inclusions and the matrix. More precisely, in accordance to the first law of thermodynamics we allow that some thermal flux - in fact the difference of the incoming and outgoing thermal fluxes - is accumulated across the interface. And we assume that it is proportional to the speed of change of the thermal flux within the tangent direction.

It is worth mentioning that our boundary value problem can be used for the characterization of other physical or mechanical processes. The analogous boundary contact conditions known as "membrane type" interface conditions can be found in classical problems of solid mechanics for elastic media. For more details, we refer to [3] where different types of boundary contact conditions are described.

In addition, we assume that the heat flux is periodic on $y$. Thus,

$$
\lambda_{m} \frac{\partial T_{m}}{\partial y}\left(x, \frac{1}{2}\right)=\lambda_{m} \frac{\partial T_{m}}{\partial y}\left(x,-\frac{1}{2}\right)=-A \sin \theta+q_{1}(x),
$$

where $A$ is the intensity of an external flux. The heat flux is also periodic on $x$, and, consequently,

$$
\lambda_{m} \frac{\partial T_{m}}{\partial x}\left(-\frac{1}{2}, y\right)=\lambda_{m} \frac{\partial T_{m}}{\partial x}\left(\frac{1}{2}, y\right)=-A \cos \theta+q_{2}(y)
$$


To complement the average flux conditions at infinity, the latter immediately proves that the equalities

$$
\int_{-1 / 2}^{1 / 2} q_{j}(\xi) d \xi=0
$$

are valid for the unknown functions $q_{j}(j=1,2)$. The unknown functions $q_{1}$ and $q_{2}$ mean that the flux in the boundaries of the cell is not constant, but on the assumption of source or sink absence inside the cell it should disappear in average. As a result of (2.4) and (2.5), the heat flux has a zero mean value along the boundary of the cell

$$
\int \frac{\partial T_{m}(s)}{\partial n} d s=0, \quad \int_{\partial Q_{\left(m_{1}, m_{2}\right)}+m_{1}+\imath m_{2}} \frac{\partial T_{m}(s)}{\partial n} d s=0 .
$$

The condition (2.6) is a consequence of the fact that no source (sink) exists in the cells.

\section{Solvability result and effective properties}

We will solve the problem (2.1)-(2.6) using the same approach as in [5]. We shall now outline some basic facts which we apply.

Let us introduce complex potentials $\varphi(z)$ and $\varphi_{k}(z)$ which are analytic in $D_{0}$ and $D_{k}$, and continuously differentiable in the closures of $D_{0}$ and $D_{k}$, respectively, by using the following relations

$$
T(z)=\left\{\begin{array}{l}
\operatorname{Re}(\varphi(z)+B z), z \in D_{\text {matrix }} \\
\frac{2 \lambda_{m}}{\lambda_{m}+\lambda_{k}} \operatorname{Re} \varphi_{k}(z), z \in D_{\text {inc }}
\end{array}\right.
$$

where $B$ is an unknown constant belonging to $\mathbb{C}$. Besides, we assume that the real part of $\varphi$ is doubly periodic in $D_{0}$, i.e.,

$$
\operatorname{Re} \varphi(z+1)-\operatorname{Re} \varphi(z)=0, \quad \operatorname{Re} \varphi(z+\imath)-\operatorname{Re} \varphi(z)=0 .
$$

It is shown in [13] that $\varphi$ is a single-valued function in $D_{\text {matrix }}$. The harmonic conjugate to $T$ is a function $v$ which has the following form:

$$
v(z)=\left\{\begin{array}{l}
\operatorname{Im}(\varphi(z)+B z), z \in D_{\text {mtrix }} \\
\frac{2 \lambda_{m}}{\lambda_{m}+\lambda_{k}} \operatorname{Im} \varphi_{k}(z), z \in D_{\text {inc }}
\end{array}\right.
$$

with the same unknown constant $B$.

For the determination of the flux $\nabla T(x, y)$, we introduce the derivatives of the complex potentials:

$$
\begin{array}{ll}
\psi(z):=\frac{\partial \varphi}{\partial z}=\frac{\partial T_{m}}{\partial x}-\imath \frac{\partial T_{m}}{\partial y}-B, & z \in D_{0}, \\
\psi_{k}(z):=\frac{\partial \varphi_{k}}{\partial z}=\frac{\lambda_{m}+\lambda_{k}}{2 \lambda_{m}}\left(\frac{\partial T_{k}}{\partial x}-\imath \frac{\partial T_{k}}{\partial y}\right), & z \in D_{k} .
\end{array}
$$


As it was shown in [5], the boundary value of the normal derivative can be written in the form

$$
\begin{aligned}
\frac{\partial T_{k}(t)}{\partial n} & =\operatorname{Re}\left(\left(n_{1}+\imath n_{2}\right)\left(\frac{\partial T_{k}}{\partial x}-\imath \frac{\partial T_{k}}{\partial y}\right)\right) \\
& =\frac{2 \lambda_{m}}{r_{k}\left(\lambda_{m}+\lambda_{i}\right)} \operatorname{Re}\left[\left(t-a_{k}\right)\left(\varphi_{k}\right)^{\prime}(t)\right]
\end{aligned}
$$

and the boundary value of the tangent derivative is

$$
\begin{aligned}
\frac{\partial T_{k}(t)}{\partial s} & =\operatorname{Im}\left(-\left(n_{1}+\imath n_{2}\right)\left(\frac{\partial T_{k}}{\partial x}-\imath \frac{\partial T_{k}}{\partial y}\right)\right) \\
& =-\frac{2 \lambda_{m}}{r_{k}\left(\lambda_{m}+\lambda_{i}\right)} \operatorname{Im}\left[\left(t-a_{k}\right)\left(\varphi_{k}\right)^{\prime}(t)\right] .
\end{aligned}
$$

Applying the Cauchy-Riemann equations $\frac{\partial T_{m}}{\partial n}=\frac{\partial v_{m}}{\partial s}, \frac{\partial T_{k}}{\partial n}=\frac{\partial v_{k}}{\partial s}$ the equality (2.3) can be written as

$$
\lambda_{m} \frac{\partial v_{m}}{\partial s}(t)-\lambda_{k} \frac{\partial v_{k}}{\partial s}(t)=\beta_{k} \frac{\partial^{2} T_{k}}{\partial s^{2}}, \quad\left|t-a_{k}\right|=r_{k}
$$

Integrating the last equality on $s$, we arrive at the relation

$$
\lambda_{m} v_{m}(t)-\lambda_{k} v_{k}(t)=\beta_{k} \frac{\partial T_{k}}{\partial s}+c
$$

where $c$ is an arbitrary constant. We put $c=0$ since the imaginary part of the function $\varphi$ is determined up to an additive constant which does not impact on the form of $T$. Using (3.2) and (3.3), we have

$$
\operatorname{Im} \varphi(t)=-\operatorname{Im} B t+\frac{2 \lambda_{k}}{\lambda_{m}+\lambda_{k}} \operatorname{Im} \varphi_{k}(t)-\frac{2 \beta_{k}}{r_{k}\left(\lambda_{m}+\lambda_{k}\right)} \operatorname{Im}\left[\left(t-a_{k}\right)\left(\varphi_{k}\right)^{\prime}(t)\right] .
$$

Using (3.1), we are able to write the equality (2.2) in the following form:

$$
\operatorname{Re} \varphi(t)=-\operatorname{Re} B t+\frac{2 \lambda_{m}}{\lambda_{m}+\lambda_{m}} \operatorname{Re} \varphi_{k}+\frac{2 \lambda_{m} \lambda_{k}}{\gamma_{k} r_{k}\left(\lambda_{m}+\lambda_{k}\right)} \operatorname{Re}\left[\left(t-a_{k}\right)\left(\varphi_{k}\right)^{\prime}(t)\right]
$$

Adding the relation (3.5) and (3.4) multiplied by $\imath$, and using $\operatorname{Re} \varphi_{k}=\frac{\varphi_{k}+\overline{\varphi_{k}}}{2}$, $\operatorname{Im} \varphi_{k}=\frac{\varphi_{k}-\overline{\varphi_{k}}}{2 \imath}, t-a_{k}=\frac{r_{k}^{2}}{\overline{t-a_{k}}}$, we rewrite the conditions (2.2) and (2.3) in terms of the complex potentials $\varphi(z)$ and $\varphi_{k}(z)$ :

$$
\varphi(t)=\varphi_{k}(t)-\rho_{k} \overline{\varphi_{k}(t)}+\mu_{k}\left(t-a_{k}\right)\left(\varphi_{k}\right)^{\prime}(t)+\eta_{k} \frac{r_{k}^{2}}{t-a_{k}} \overline{\left(\varphi_{k}\right)^{\prime}(t)}-B t,
$$

where

$$
\rho_{k}=\frac{\lambda_{k}-\lambda_{m}}{\lambda_{m}+\lambda_{k}}, \mu_{k}=\frac{\lambda_{m} \lambda_{k}-\beta_{k} \gamma_{k}}{r_{k} \gamma_{k}\left(\lambda_{m}+\lambda_{k}\right)}, \eta_{k}=\frac{\lambda_{m} \lambda_{k}+\beta_{k} \gamma_{k}}{r_{k} \gamma_{k}\left(\lambda_{m}+\lambda_{k}\right)}
$$


Representing the function $\varphi_{k}$ in the form $\varphi_{k}(z)=\sum_{l=0}^{\infty} \alpha_{k}\left(z-a_{k}\right)^{l},\left|z-a_{k}\right| \leq r_{k}$, and by using the relation $t=\frac{r_{k}^{2}}{t-a_{k}}+a_{k}$ on the boundary $\left|t-a_{k}\right|=r_{k}$, one get

$$
[\overline{\varphi(t)}]^{\prime}=-\left(\frac{r_{k}}{t-a_{k}}\right)^{2} \overline{\varphi^{\prime}(t)}, \quad\left[\overline{\varphi^{\prime}(t)}\right]^{\prime}=-\left(\frac{r_{k}}{t-a_{k}}\right)^{2} \overline{\psi^{\prime}(t)}
$$

Thus, after differentiating (3.6), we arrive at the following $R$-linear boundary value problem on each contour $\left|t-a_{k}\right|=r_{k}$ :

$$
\begin{aligned}
\psi(t)= & \left(1+\mu_{k}\right) \psi_{k}(t)+\left(\rho_{k}-\eta_{k}\right)\left(\frac{r_{k}}{t-a_{k}}\right)^{2} \overline{\psi_{k}(t)}+\mu_{k}\left(t-a_{k}\right) \psi_{k}^{\prime}(t) \\
& -\frac{\eta_{k} r_{k}^{4}}{\left(t-a_{k}\right)^{3}} \overline{\psi_{k}^{\prime}(t)}-B
\end{aligned}
$$

with the unknown constant $B$.

The problem (3.8) is solved in [5] with small changes in real coefficients. Note that the parameters $\rho_{k}, \mu_{k}$ defined in (3.7) satisfy the inequalities $\left|\rho_{k}\right|<1$ and $\mu_{k}>0$, for which all solvability theorems stated in [5] hold true.

In order to find the functions $\psi$ and $\psi_{k}$ inside the matrix and inclusions, respectively, we need some of the results from [6].

Let the functions $\widetilde{\psi}^{(1)}$ and $\widetilde{\psi}_{k}^{(1)}$ be solutions of an auxiliary problem (2.1)(2.3) with a constant jump corresponding to the external field applied in the $x$-direction

$$
T(z+1)=T(z)+1, \quad T(z+\imath)=T(z)
$$

(instead of conditions $(2.4),(2.5))$ corresponding to the following $R$-linear conjugation boundary value problem

$$
\begin{aligned}
\widetilde{\psi}^{(1)}(t)= & \left(1+\mu_{k}\right) \widetilde{\psi}_{k}^{(1)}(t)+\left(\rho_{k}-\eta_{k}\right)\left(\frac{r_{k}}{t-a_{k}}\right)^{2} \overline{\widetilde{\psi}_{k}^{(1)}(t)} \\
& +\mu_{k}\left(t-a_{k}\right)\left(\widetilde{\psi}_{k}^{(1)}(t)\right)^{\prime}-\eta_{k} \frac{r_{k}^{4}}{\left(t-a_{k}\right)^{3}} \overline{\left(\widetilde{\psi}_{k}^{(1)}(t)\right)^{\prime}}-1,
\end{aligned}
$$

and let functions $\widetilde{\psi}^{(2)}$ and $\widetilde{\psi}_{k}^{(2)}$ be solutions of an auxiliary problem (2.1)(2.3) with a constant jump corresponding to the external field applied in the $y$-direction

$$
T(z+1)=T(z), \quad T(z+\imath)=T(z)-1
$$

(instead of conditions $(2.4),(2.5))$ corresponding to the problem

$$
\begin{aligned}
\widetilde{\psi}^{(2)}(t)= & \left(1+\mu_{k}\right) \widetilde{\psi}_{k}^{(2)}(t)+\left(\rho_{k}-\eta_{k}\right)\left(\frac{r_{k}}{t-a_{k}}\right)^{2} \overline{\widetilde{\psi}_{k}^{(2)}(t)} \\
& +\mu_{k}\left(t-a_{k}\right)\left(\widetilde{\psi}_{k}^{(2)}(t)\right)^{\prime}-\eta_{k} \frac{r_{k}^{4}}{\left(t-a_{k}\right)^{3}} \overline{\left(\widetilde{\psi}_{k}^{(2)}(t)\right)^{\prime}}-\imath .
\end{aligned}
$$


The functions $\widetilde{\psi}^{\perp}(z):=\imath \widetilde{\psi}^{(2)}(\imath z)$ and $\widetilde{\psi}_{k}^{\perp}(z):=\imath \widetilde{\psi}_{k}^{(2)}(\imath z)$ satisfy the following $R$-linear conjugation boundary value problem

$$
\begin{aligned}
\widetilde{\psi}^{\perp}(t)= & \left(1+\mu_{k}\right) \widetilde{\psi}_{k}^{\perp}(t)+\left(\rho_{k}-\eta_{k}\right)\left(\frac{r_{k}}{t-b_{k}}\right)^{2} \overline{\widetilde{\psi}_{k}^{\perp}(t)} \\
& +\mu_{k}\left(t-b_{k}\right)\left(\widetilde{\psi}_{k}^{\perp}(t)\right)^{\prime}-\eta_{k} \frac{r_{k}^{4}}{\left(t-b_{k}\right)^{3}} \overline{\left(\widetilde{\psi}_{k}^{\perp}(t)\right)^{\prime}}+1,
\end{aligned}
$$

where $\left|t-b_{k}\right|=r_{k}, b_{k}=-\imath a_{k}$. We introduce

$$
I:=\int_{-\frac{1}{2}}^{\frac{1}{2}} \operatorname{Re} \widetilde{\psi}^{(1)}\left(\frac{1}{2}+\imath y\right) d y, \quad I^{\perp}:=\int_{-\frac{1}{2}}^{\frac{1}{2}} \operatorname{Re} \widetilde{\psi}^{\perp}\left(\frac{1}{2}+\imath y\right) d y .
$$

Then, by using the same techniques as in [6], the following results hold.

Theorem 1. Let $T_{m}=T_{m}(x, y)$ and $T_{k}=T_{k}(x, y)$ be the solution of the problem (2.2)-(2.3), (2.4) and (2.5). The temperature distribution can be found up to an arbitrary constant and is defined in the form (3.1), where

$$
\begin{aligned}
B & =\frac{-A \cos \theta}{\lambda_{m}(I+1)}-\frac{A \sin \theta}{\lambda_{m}\left(I^{\perp}-1\right)} \imath, \\
\varphi(z) & =\frac{-A \cos \theta}{\lambda_{m}(I+1)} \widetilde{\varphi}^{(1)}(z)-\frac{A \sin \theta}{\lambda_{m}\left(I^{\perp}-1\right)} \widetilde{\varphi}^{\perp}(-\imath z), \\
\varphi_{k}(z) & =\frac{-A \cos \theta}{\lambda_{m}(I+1)} \widetilde{\varphi}_{k}^{(1)}(z)-\frac{A \sin \theta}{\lambda_{m}\left(I^{\perp}-1\right)} \widetilde{\varphi}_{k}^{\perp}(-\imath z) .
\end{aligned}
$$

Theorem 2. Let $T_{m}=T_{m}(x, y)$ and $T_{k}=T_{k}(x, y)$ be the solution of the problem (2.2)-(2.3), (2.4) and (2.5). The temperature flux is defined in the following form:

$$
\frac{\partial T(x, y)}{\partial x}-\imath \frac{\partial T(x, y)}{\partial y}=\left\{\begin{array}{l}
\psi(z)+B, z=x+\imath y \in D_{\text {matrix }} \\
\frac{2 \lambda_{m}}{\lambda_{m}+\lambda_{k}} \psi_{k}(z), z=x+\imath y \in D_{i n c}
\end{array}\right.
$$

with

$$
B=\frac{-A \cos \theta}{\lambda_{m}(I+1)}-\frac{A \sin \theta}{\lambda_{m}\left(I^{\perp}-1\right)} \imath,
$$

and

$$
\begin{aligned}
\psi(z) & :=\frac{-A \cos \theta}{\lambda_{m}(I+1)} \widetilde{\psi}^{(1)}(z)+\imath \frac{A \sin \theta}{\lambda_{m}\left(I^{\perp}-1\right)} \widetilde{\psi}^{\perp}(-\imath z), \quad z \in D_{\text {matrix }}, \\
\psi_{k}(z) & :=\frac{-A \cos \theta}{\lambda_{m}(I+1)} \widetilde{\psi}_{k}^{(1)}(z)+\imath \frac{A \sin \theta}{\lambda_{m}\left(I^{\perp}-1\right)} \widetilde{\psi}_{k}^{\perp}(-\imath z), \quad z \in D_{\text {inc }},
\end{aligned}
$$

where the functions $\widetilde{\psi}^{(1)}, \widetilde{\psi}^{\perp}, \widetilde{\psi}_{k}^{(1)}$ and $\widetilde{\psi}_{k}^{\perp}$ are derivatives of the functions $\widetilde{\varphi}^{(1)}$, $\widetilde{\varphi}^{\perp}, \widetilde{\varphi}_{k}^{(1)}$ and $\widetilde{\varphi}_{k}^{\perp}$, respectively. 
The proofs of these theorems are direct consequences of the results obtained in [6], where an analogous to (3.6), (3.8) problems are analytically solved using the method of functional equations. Here, we use some facts and notations of the paper [6] to show briefly how to find the functions $\widetilde{\psi}^{(1)}$ and $\widetilde{\psi}_{k}^{(1)}$ contained in (3.9). The functions $\widetilde{\psi}^{\perp}$ and $\widetilde{\psi}_{k}^{\perp}$ can be analogously found.

Notice that we have $N$ contours $\partial D_{k}$ and $N$ complex conjugation conditions on each contour $\partial D_{k}$ but we need to find $N+1$ functions $\widetilde{\psi}^{(1)}, \widetilde{\psi}_{1}^{(1)}, \ldots, \widetilde{\psi}_{N}^{(1)}$. This means that we need one additional condition to close up the system. For this reason we introduce a new doubly periodic function $\Phi$ which is sectionally analytic in $Q_{(0,0)}$ and in $\bigcup_{k=1}^{N} D_{k}$ and has zero jumps along each $\partial D_{k}, k=$ $1,2, \ldots, N$. Such consideration will give an additional condition on $\widetilde{\psi}^{(1)}, \widetilde{\psi}_{k}^{(1)}$. In fact, we will show that $\Phi \equiv 0$.

Let us introduce the sectionally analytic doubly periodic function $\Phi$ by the following formula:

$$
\Phi(z)=\left\{\begin{array}{l}
\Phi_{(k)}(z),\left|z-a_{k}\right| \leq r_{k}, \\
\Phi_{(0)}(z), z \in D_{0},
\end{array}\right.
$$

where

$$
\begin{aligned}
& \Phi_{(k)}(z)=\left(1+\mu_{k}\right) \widetilde{\psi}_{k}^{(1)}(z)+\mu_{k}\left(z-a_{k}\right)\left(\widetilde{\psi}_{k}^{(1)}\right)^{\prime}(z)-\sum_{m=1}^{N} \sum_{m_{1}, m_{2}} *\left(\rho_{m}-\eta_{m}\right) \\
& \times W_{m_{1}, m_{2}, m} \widetilde{\psi}_{m}^{(1)}(z)+\sum_{m=1}^{N} \sum_{m_{1}, m_{2}}^{*} \eta_{m} W_{m_{1}, m_{2}, m}^{\prime}\left(\widetilde{\psi}_{m}^{(1)}\right)^{\prime}(z)-1, \\
& \Phi_{(0)}(z)=\widetilde{\psi}^{(1)}(z)-\sum_{m=1}^{N} \sum_{m_{1}, m_{2}}\left(\rho_{m}-\eta_{m}\right) W_{m_{1}, m_{2}, m} \widetilde{\psi}_{m}^{(1)}(z) \\
& +\sum_{m=1}^{N} \sum_{m_{1}, m_{2}} \eta_{m} W_{m_{1}, m_{2}, m}^{\prime}\left(\widetilde{\psi}_{m}^{(1)}\right)^{\prime}(z) .
\end{aligned}
$$

Here,

$$
\begin{aligned}
& W_{m_{1}, m_{2}, k} \widetilde{\psi}_{k}^{(1)}(z)=\left(\frac{r_{k}}{z-a_{k}-m_{1}-\imath m_{2}}\right)^{2} \overline{\widetilde{\psi}_{k}^{(1)}\left(\frac{r_{k}^{2}}{\overline{z-a_{k}-m_{1}-\imath m_{2}}}+a_{k}\right)}, \\
& W_{m_{1}, m_{2}, k}^{\prime}\left(\widetilde{\psi}_{k}^{(1)}\right)^{\prime}(z)=\frac{r_{k}^{4}}{\left(z-a_{k}-m_{1}-\imath m_{2}\right)^{3}} \overline{\left(\widetilde{\psi}_{k}^{(1)}\right)^{\prime}\left(\frac{r_{k}^{2}}{\overline{z-a_{k}-m_{1}-\imath m_{2}}}+a_{k}\right)} \text {, } \\
& \sum_{m=1}^{N} \sum_{m_{1}, m_{2}}^{*}\left(\rho_{m}-\eta_{m}\right) W_{m_{1}, m_{2}, m}:=\sum_{m \neq k} \sum_{m_{1}, m_{2}}\left(\rho_{m}-\eta_{m}\right) W_{m_{1}, m_{2}, m} \\
& +\sum_{m_{1}, m_{2}}\left(\rho_{k}-\eta_{k}\right) W_{m_{1}, m_{2}, k}
\end{aligned}
$$


The "prime" notation in $\sum_{m_{1}, m_{2}}$ ' means that the summation occurs in all $m_{1}$ and $m_{2}$ except at $\left(m_{1}, m_{2}\right)=(0,0)$.

Applying the Analytic Continuation Principle and Liouville's theorem for doubly periodic functions, we have that $\Phi=c$. Besides, it is possible to show that $\Phi=0$ (cf. [6]). Writing $\Phi(z) \equiv 0$, we obtain the following system of linear functional equations

$$
\begin{gathered}
\left.\widetilde{\psi}_{k}^{(1)}(z)=-\frac{\mu_{k}}{1+\mu_{k}}\left(z-a_{k}\right) \widetilde{\left(\psi_{k}^{(1)}\right.}\right)^{\prime}(z)+\frac{1}{1+\mu_{k}} \sum_{m=1}^{N} \sum_{j}^{*}\left(\rho_{m}-\eta_{m}\right) \\
\left.\times W_{j, m} \widetilde{\psi_{m}^{(1)}}(z)-\frac{1}{1+\mu_{k}} \sum_{m=1}^{N} \sum_{j}{ }^{*} \eta_{m} W_{j, m}^{\prime} \widetilde{\left(\psi_{m}^{(1)}\right.}\right)^{\prime}(z)+\frac{1}{1+\mu_{k}}
\end{gathered}
$$

(for $k=1,2, \ldots, N$ ) which is uniquely solvable with respect to $\widetilde{\psi}_{k}^{(1)}$ in the space of analytical functions (for more details cf. [7]).

The function $\widetilde{\psi}^{(1)}$ has the form

$$
\widetilde{\psi}^{(1)}(z)=\sum_{m=1}^{N} \sum_{j}\left(\rho_{m}-\eta_{m}\right) W_{j, m} \widetilde{\psi}_{m}^{(1)}(z)-\sum_{m=1}^{N} \sum_{j} \eta_{m} W_{j, m}^{\prime} \widetilde{\left(\psi_{m}^{(1)}\right)^{\prime}}(z) .
$$

Let us expand $\widetilde{\psi}_{k}^{(1)}(z)$ into Taylor series,

$$
\widetilde{\psi}_{k}^{(1)}(z)=\sum_{l=0}^{\infty} \widetilde{\psi}_{l k}^{(1)}\left(z-a_{k}\right)^{l}, \quad\left(\widetilde{\psi}_{k}^{(1)}\right)^{\prime}(z)=\sum_{l=1}^{\infty} \widetilde{\psi}_{l k}^{(1)} l\left(z-a_{k}\right)^{l-1},
$$

in order to sum up $W_{m_{1}, m_{2}, k} \widetilde{\psi}_{k}^{(1)}(z)$ and $W_{m_{1}, m_{2}, k}^{\prime}\left(\widetilde{\psi}_{k}^{(1)}\right)^{\prime}(z)$ over all translations $m_{1}+\imath m_{2}$.

The series $\sum_{j} W_{j, k} \widetilde{\psi}_{k}^{(1)}(z)$, where $j=\left(m_{1}, m_{2}\right)$ and $k$ is a fixed number, can be represented via elliptic Eisenstein functions $E_{l}(z)$ of order $l$ (see $[7,19]$ ):

$$
\begin{gathered}
\sum_{j} W_{j, k} \widetilde{\psi}_{k}^{(1)}(z)=\sum_{l=0}^{\infty} \overline{\widetilde{\psi}_{l k}^{(1)}} r_{k}^{2(l+1)} E_{l+2}\left(z-a_{k}\right), \\
\sum_{j} W_{j, k}^{\prime}\left(\widetilde{\psi}_{k}^{(1)}\right)^{\prime}(z)=\sum_{l=1}^{\infty} \overline{\widetilde{\psi}_{l k}^{(1)}} l r_{k}^{2(l+1)} E_{l+2}\left(z-a_{k}\right) .
\end{gathered}
$$

The series

$$
\sum_{j}{ }^{\prime} W_{j, k} \widetilde{\psi}_{k}^{(1)}(z):=\sum_{j} W_{j, k} \widetilde{\psi}_{k}^{(1)}(z)-\left(\frac{r_{k}}{z-a_{k}}\right)^{2} \overline{\widetilde{\psi}_{k}^{(1)}\left(\frac{r_{k}^{2}}{\overline{z-a_{k}}}+a_{k}\right)}
$$

and

$$
\sum_{j}{ }^{\prime} W_{j, k}^{\prime}\left(\widetilde{\psi}_{k}^{(1)}\right)^{\prime}(z):=\sum_{j} W_{j, k}^{\prime}\left(\widetilde{\psi}_{k}^{(1)}\right)^{\prime}(z)-\frac{r_{k}^{4}}{\left(z-a_{k}\right)^{3}} \overline{\left(\widetilde{\psi}_{k}^{(1)}\right)^{\prime}\left(\frac{r_{k}^{2}}{\overline{z-a_{k}}}+a_{k}\right)}
$$


can be written in the form

$$
\begin{aligned}
& \sum_{j}{ }^{\prime} W_{j, k} \widetilde{\psi}_{k}^{(1)}(z)=\sum_{l=0}^{\infty} \overline{\widetilde{\psi}_{l k}^{(1)}} r_{k}^{2(l+1)} \sigma_{l+2}\left(z-a_{k}\right), \\
& \sum_{j}{ }^{\prime} W_{j, k}^{\prime}\left(\widetilde{\psi}_{k}^{(1)}\right)^{\prime}(z)=\sum_{l=1}^{\infty} \overline{\widetilde{\psi}_{l k}^{(1)}} l r_{k}^{2(l+1)} \sigma_{l+2}\left(z-a_{k}\right),
\end{aligned}
$$

where $\sigma_{l}$ is the modified Eisenstein function defined by the formula $\sigma_{l}(z):=$ $E_{l}(z)-z^{-l}$. The Eisenstein functions $E_{l}$ converge absolutely and uniformly for $l=3,4, \ldots$ and conditionally for $l=2$ (cf. [19]).

Thus, we can rewrite the equations $(3.10)$ and $(3.11)$, for $\widetilde{\psi}_{k}^{(1)}$ and $\widetilde{\psi}^{(1)}$, as follows:

$$
\begin{aligned}
& \widetilde{\psi}_{k}^{(1)}(z)=-\frac{\mu_{k}}{1+\mu_{k}}\left(z-a_{k}\right)\left(\widetilde{\psi}_{k}^{(1)}\right)^{\prime}(z)+\frac{1}{1+\mu_{k}} \sum_{m \neq k}^{N} \sum_{l=0}^{\infty}\left(\rho_{m}-\eta_{m}\right) \\
& \times \overline{\widetilde{\psi}_{l m}^{(1)}} r_{m}^{2(l+1)} E_{l+2}\left(z-a_{m}\right)+\frac{\rho_{k}-\eta_{k}}{1+\mu_{k}} \sum_{l=0}^{\infty} \overline{\widetilde{\psi}_{l k}^{(1)}} r_{k}^{2(l+1)} \sigma_{l+2}\left(z-a_{k}\right) \\
& -\frac{1}{1+\mu_{k}} \sum_{m \neq k}^{N} \sum_{l=1}^{\infty} \eta_{m} \overline{\widetilde{\psi}_{l m}^{(1)}} l r_{m}^{2(l+1)} E_{l+2}\left(z-a_{m}\right) \\
& -\frac{\eta_{k}}{1+\mu_{k}} \sum_{l=1}^{\infty} \overline{\widetilde{\psi}_{l k}^{(1)}} l r_{k}^{2(l+1)} \sigma_{l+2}\left(z-a_{k}\right)+\frac{1}{1+\mu_{k}} \\
& \widetilde{\psi}^{(1)}(z)=\sum_{m=1}^{N} \sum_{l=0}^{\infty}\left(\rho_{m}-\eta_{m}\right) \overline{\widetilde{\psi}_{l m}^{(1)}} r_{m}^{2(l+1)} E_{l+2}\left(z-a_{m}\right) \\
& -\sum_{m=1}^{N} \sum_{l=1}^{\infty} \eta_{m} \overline{\widetilde{\psi}_{l m}^{(1)}} l r_{m}^{2(l+1)} E_{l+2}\left(z-a_{m}\right) .
\end{aligned}
$$

Using (3.12), we have

$$
\widetilde{\psi}_{k}^{(1)}(z)+\frac{\mu_{k}}{1+\mu_{k}}\left(z-a_{k}\right)\left(\widetilde{\psi}_{k}^{(1)}\right)^{\prime}(z)=\sum_{l=0}^{\infty} \widetilde{\psi}_{l k}^{(1)}\left(z-a_{k}\right)^{l}\left(1+\frac{l \mu_{k}}{1+\mu_{k}}\right) .
$$

Now we need to find the numerical coefficients $\widetilde{\psi}_{l m}^{(1)}$ of the system (3.13). Note that the equation (3.14) for $\widetilde{\psi}^{(1)}$ has the same coefficients $\widetilde{\psi}_{l m}^{(1)}$. Taking a partial sum of the Taylor series with the first $M$ terms

$$
\widetilde{\psi}_{k}^{(1)}(z)=\widetilde{\psi}_{0 k}^{(1)}+\widetilde{\psi}_{1 k}^{(1)}\left(z-a_{k}\right)+\widetilde{\psi}_{2 k}^{(1)}\left(z-a_{k}\right)^{2}+\cdots+\widetilde{\psi}_{M k}^{(1)}\left(z-a_{k}\right)^{M}
$$

and collecting the coefficients of the consequent powers of $z-a_{k}$, we obtain the formula for the definition of $\widetilde{\psi}_{j k}^{(1)}$ :

$$
\widetilde{\psi}_{j k}^{(1)}=\left.\frac{1+\mu_{k}}{j !\left(1+(1+j) \mu_{k}\right)} \widetilde{\psi}_{k}^{(j)}\right|_{z=a_{k}}
$$

where $\widetilde{\psi}_{k}^{(j)}$ is the derivative of order $j$ of the function $\widetilde{\psi}_{k}^{(1)}$. 
Theorem 3. For the parameters $\mu_{k}>0, k=1, \ldots, N$, the equation (3.13) has a unique solution in $\mathcal{H}^{2}$.

Here, $\mathcal{H}^{2}\left(D_{k}\right)$ is the generalized Hardy space of analytic functions on $D_{k}$ satisfying the condition

$$
\sup _{0<r<r_{k}} \int_{0}^{2 \pi}\left|\psi_{k}\left(r e^{\imath \theta}+a_{k}\right)\right|^{2} d \theta<\infty
$$

and endowed with the norm

$$
\left\|\psi_{k}\right\|_{\mathcal{H}^{2}\left(D_{k}\right)}^{2}:=\sup _{0<r<r_{k}} \int_{0}^{2 \pi}\left|\psi_{k}\left(r e^{\imath \theta}+a_{k}\right)\right|^{2} d \theta .
$$

As far as the authors know, the first results devoted to functional equations in Hardy spaces appeared in [9] and [8]. The ideas of these papers were applied to modified equations in [7], where one can find the proof of the Theorem 3.

Remark 1. It directly follows from Theorem 3 and (3.7) that the system (3.13), (3.14) always has a unique solution, as since $\gamma_{k}>0$ and $\beta_{k}<0$, according to the statement of the problem.

Now the components of the effective conductivity tensor

$$
\Lambda_{e}=\left(\begin{array}{cc}
\lambda_{e}^{x} & \lambda_{e}^{x y} \\
\lambda_{e}^{x y} & \lambda_{e}^{y}
\end{array}\right)
$$

can be found from the well-known equation

$$
\langle\mathbf{q}\rangle=-\Lambda_{e} \cdot\langle\nabla T\rangle
$$

where $\langle\mathbf{q}\rangle=\left(\mathfrak{q}_{1}, \mathfrak{q}_{2}\right)$ is the average flux, and $\langle\nabla T\rangle=\left(T_{1}, T_{2}\right)$ is the average temperature gradient with

$$
\begin{aligned}
\mathfrak{q}_{1} & =\lambda_{m} \iint_{D_{0}} \frac{\partial T_{m}}{\partial x} d x d y+\sum_{k=1}^{N} \lambda_{k} \iint_{D_{k}} \frac{\partial T_{k}}{\partial x} d x d y, \\
\mathfrak{q}_{2} & =\lambda_{m} \iint_{D_{0}} \frac{\partial T_{m}}{\partial y} d x d y+\sum_{k=1}^{N} \lambda_{k} \iint_{D_{k}} \frac{\partial T_{k}}{\partial y} d x d y, \\
T_{1} & =\iint_{D_{0}} \frac{\partial T_{m}}{\partial x} d x d y+\sum_{k=1}^{N} \iint_{D_{k}} \frac{\partial T_{k}}{\partial x} d x d y, \\
T_{2} & =\iint_{D_{0}} \frac{\partial T_{m}}{\partial y} d x d y+\sum_{k=1}^{N} \iint_{D_{k}} \frac{\partial T_{k}}{\partial y} d x d y .
\end{aligned}
$$


Thus, by using the same arguments as in [5], we derive

$$
\begin{aligned}
\mathfrak{q}_{1}= & \lambda_{m}\left(\oint_{\partial Q_{(0,0)}} x \frac{\partial T_{m}}{\partial n} d s-\sum_{k=1}^{N} \oint_{\partial D_{k}} x \frac{\partial T_{m}}{\partial n} d s\right)+\sum_{k=1}^{N} \lambda_{k} \oint_{\partial D_{k}} x \frac{\partial T_{k}}{\partial n} d s \\
= & -A \cos \theta-\sum_{k=1}^{N} \beta_{k} \oint_{\partial D_{k}} x \frac{\partial^{2} T_{k}}{\partial s^{2}} d s . \\
\mathfrak{q}_{2}= & -A \sin \theta-\sum_{k=1}^{N} \beta_{k} \oint_{\partial D_{k}} y \frac{\partial^{2} T_{k}}{\partial s^{2}} d s, \\
T_{1}- & \imath T_{2}=\frac{-A e^{-\imath \theta}}{\lambda_{m}}+2 \sum_{k=1}^{N} \frac{\lambda_{m}-\lambda_{k}}{\lambda_{m}+\lambda_{k}} \iint_{D_{k}} \psi_{k}(z) d x d y \\
& -\frac{1}{\lambda_{m}} \sum_{k=1}^{N} \beta_{k} \oint_{\partial D_{k}}(x-\imath y) \frac{\partial^{2} T_{k}}{\partial s^{2}} d s \\
= & \frac{-A e^{-\imath \theta}}{\lambda_{m}}-2 \pi \sum_{k=1}^{N} \rho_{k} r_{k}^{2} \psi_{k}\left(a_{k}\right)-\frac{1}{\lambda_{m}} \sum_{k=1}^{N} \beta_{k} \oint_{\partial D_{k}}(x-\imath y) \frac{\partial^{2} T_{k}}{\partial s^{2}} d s .
\end{aligned}
$$

\section{Numerical results and discussions}

In this section, we present numerical calculations of the material characteristics. This will be the case for temperature, flux and the effective conductivity. Here, we are using the Maple 14 software.

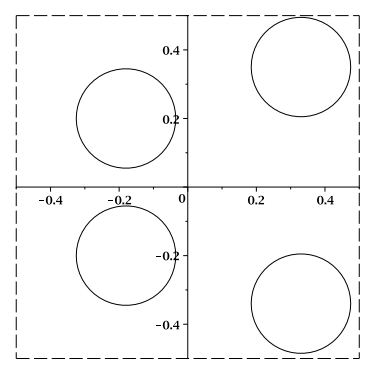

Figure 2. Configuration of the unit cell with four inclusions considered in computation.

First, we discuss the accuracy of our calculations, as well as their implementation. We choose a non-symmetrical configuration of non-overlapping inclusions with the centers

$$
a_{1}=-0.18+0.2 \imath, a_{2}=0.33-0.34 \imath, a_{3}=0.33+0.35 \imath, a_{4}=-0.18-0.2 \imath
$$

and the same radius $r_{k}=R$ of value 0.145 when the inclusions are situated very close to inclusions of the adjoin cells (see Fig. 2). It is the worst situation for accuracy in comparison with symmetrical configurations of the inclusions. 
We suppose that a heat flux of fixed intensity $A=-1$ flows in different directions with respect to the main axis depending on the angle $\theta$. The conductivity of the matrix is $\lambda_{m}=1$, and the conductivity of the inclusions $\lambda_{k}$ and parameters $\gamma_{k}, \beta_{k}$ take different values.

We calculate the temperature and flux components for different $M$, showing how many terms are selected for computations in the Taylor series (cf. (3.13) and (3.14)). Computations are given in the Table 1-2 for the first ten consecutive values of $M=0,1, \ldots, 9$ and show that taking $M=9$ the accuracy is between five or six valid units depending on where the flux is computed. Note that here, for the same configuration, we need more terms $M$ to get the same accuracy as in the case of soft or stiff imperfect contact conditions on the boundaries of material components (cf. $[5,6])$.

Table 1. Temperature in two points of the model for different numbers of $M$, while other problem parameters are: $\theta=0, \lambda_{k}=100, \gamma_{k}=0.01, \beta_{k}=-0.01$ and the configuration of the inclusions being defined by (4.1).

\begin{tabular}{lll}
\hline$M$ & $T(0)$ & $T\left(a_{1}\right)$ \\
\hline 0 & 0.072495448 & -0.320878065 \\
1 & 0.085684757 & -0.326260214 \\
2 & 0.085696009 & -0.327640325 \\
3 & 0.085538235 & -0.328182808 \\
4 & 0.085472394 & -0.328505039 \\
5 & 0.085437191 & -0.328623251 \\
6 & 0.085425520 & -0.328663509 \\
7 & 0.085421809 & -0.328677550 \\
8 & 0.085420760 & -0.328690413 \\
9 & 0.085420530 & -0.328695796 \\
\hline
\end{tabular}

Table 2. The flux components for different numbers of $M$, while other problem parameters are: $\theta=0, \lambda_{k}=100, \gamma_{k}=0.01, \beta_{k}=-0.01$ and the configuration of the inclusions being defined by (4.1).

\begin{tabular}{lllll}
\hline$M$ & $Q_{x}^{(m)}(0)$ & $Q_{y}^{(m)}(0)$ & $Q_{x}^{(1)}\left(a_{1}\right)$ & $Q_{y}^{(1)}\left(a_{1}\right)$ \\
\hline 0 & 1.509808994 & 0.007462211 & 0.004195435 & 0.000030973 \\
1 & 1.499794388 & 0.007529382 & 0.004282506 & 0.000036271 \\
2 & 1.486223434 & 0.007493638 & 0.004293984 & 0.000035871 \\
3 & 1.487420074 & 0.007613907 & 0.004294395 & 0.000036244 \\
4 & 1.488001338 & 0.007614627 & 0.004296148 & 0.000036448 \\
5 & 1.488494705 & 0.007626770 & 0.004296825 & 0.000036491 \\
6 & 1.488689358 & 0.007630776 & 0.004296991 & 0.000036510 \\
7 & 1.488753276 & 0.007632135 & 0.004297042 & 0.000036520 \\
8 & 1.488804042 & 0.007633012 & 0.004297138 & 0.000036524 \\
9 & 1.488825099 & 0.007633360 & 0.004297179 & 0.000036526 \\
\hline
\end{tabular}

We represent the temperature and flux distribution $T(x, y)$ in Figs. $3-10$ 
for the fixed parameters $R=0.145, \lambda_{m}=1$ and different $\theta=0, \frac{\pi}{4} ; \lambda_{k}=$ $100,0.01 ; \beta_{k}=-0.01,-100 ; \gamma_{k}=0.01,100$. The temperature changes faster or slower within the representative cell in dependence on the different values of the parameters $\lambda_{k}, \gamma_{k}$ and $\beta_{k}$ as it is shown in Figs. 3-6.

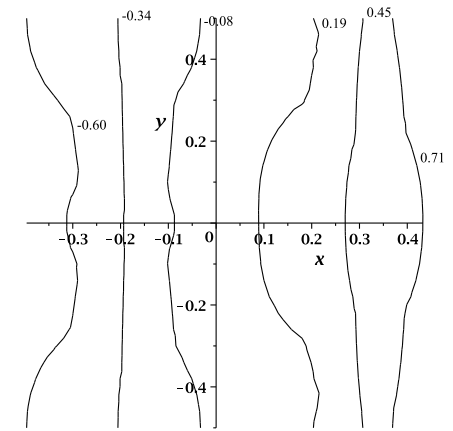

(a) $\theta=0$

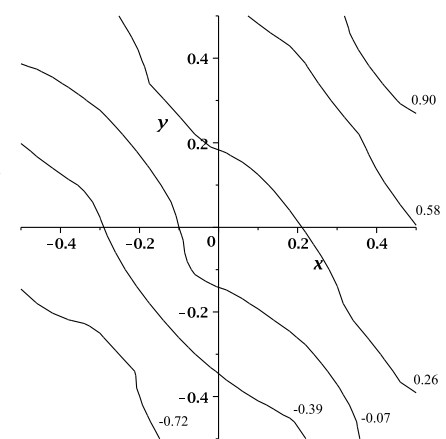

(b) $\theta=\pi / 4$

Figure 3. The temperature distribution inside $Q_{(0,0)}$ for $\lambda_{k}=0.01, \gamma_{k}=100$, $\beta_{k}=-0.01, \theta=0 ; \pi / 4$.

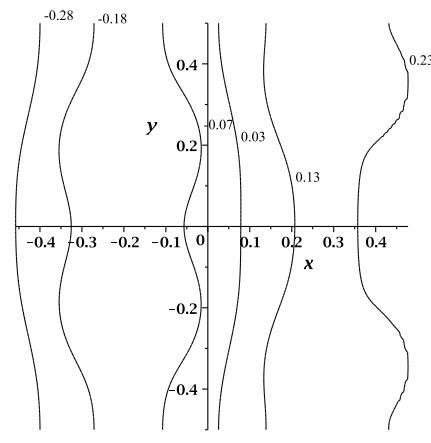

(a) $\theta=0$

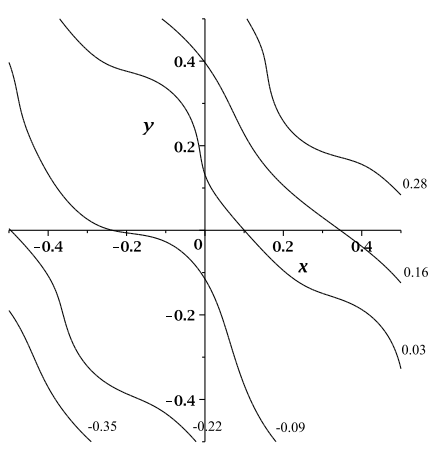

(b) $\theta=\pi / 4$

Figure 4. The temperature distribution inside $Q_{(0,0)}$ for $\lambda_{k}=100, \gamma_{k}=100$, $\beta_{k}=-0.01, \theta=0 ; \pi / 4$.

Note that if the parameter $\beta_{k}=0$, we arrive at soft imperfect contact conditions where the flux intensity in the matrix and inclusions depends not only on $\lambda_{k}$, with fixed $\lambda_{m}=1$, but also on the parameter $\gamma_{k}$ (cf. [6]). In our case of mixed imperfect contact conditions, the parameter $\beta_{k}$ also influences on the flux. For instance, it is shown in Figs. 7-10 that for fixed $\lambda_{m}=1$ and $\gamma_{k}=100$, in order to get a more intensive flux in the inclusions in comparison with the flux in the matrix, we need not only to increase $\lambda_{k}$ but also to decrease the absolute value of the parameter $\beta_{k}$. We obtain the same effect when we fix 
$\lambda_{k}=1, \gamma_{k}=100$ and decrease $\lambda_{m}$ and the absolute value of $\beta_{k}$. For all other values of the parameters $\lambda_{m}, \lambda_{k}, \gamma_{k}$ and $\beta_{k}$, the flux in the matrix is more intensive than the flux in the inclusions.

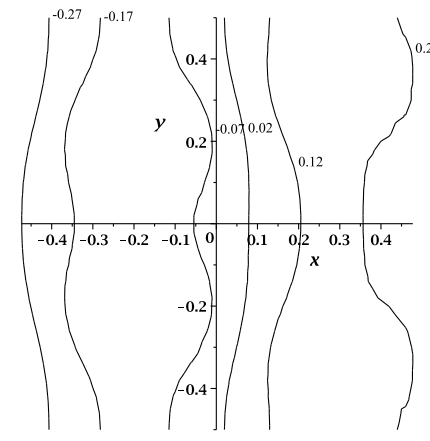

(a) $\theta=0$

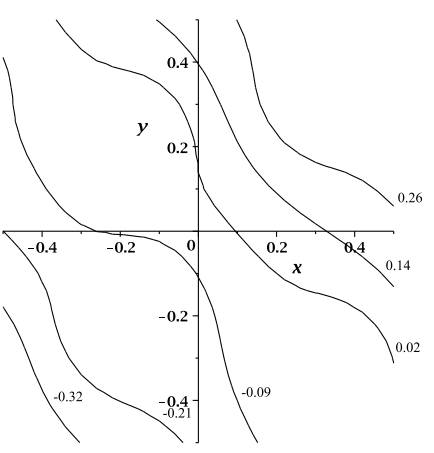

(b) $\theta=\pi / 4$

Figure 5. The temperature distribution inside $Q_{(0,0)}$ for $\lambda_{k}=0.01, \gamma_{k}=100$, $\beta_{k}=-100, \theta=0 ; \pi / 4$.

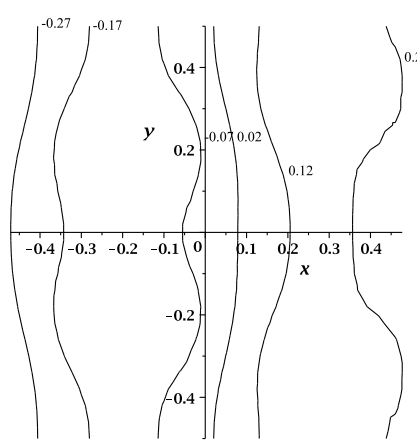

(a) $\theta=0$

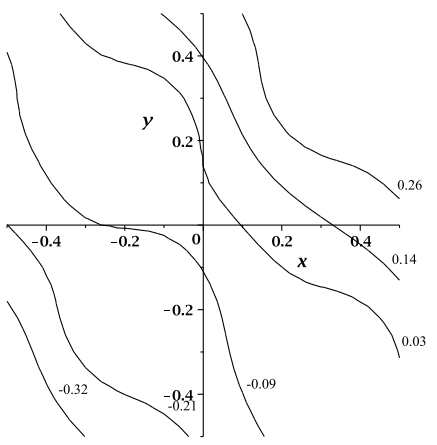

(b) $\theta=\pi / 4$

Figure 6. The temperature distribution inside $Q_{(0,0)}$ for $\lambda_{k}=100, \gamma_{k}=100, \beta_{k}=-100$, $\theta=0 ; \pi / 4$.

The values of all components of the tensor $\Lambda_{e}$ as a function on the radius $R$ are presented in Tables 3-6 for different parameters $\lambda_{k}, \gamma_{k}$ and $\beta_{k}$. The calculations were performed with the found accuracy between five or six valid units. 


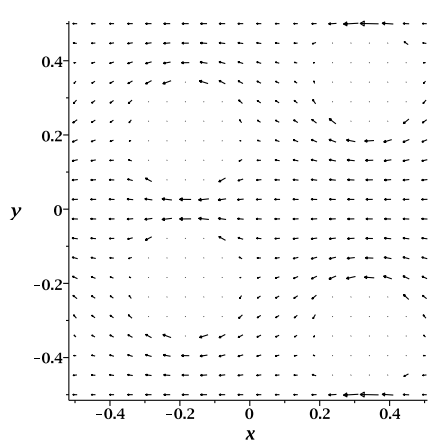

(a) $\theta=0$

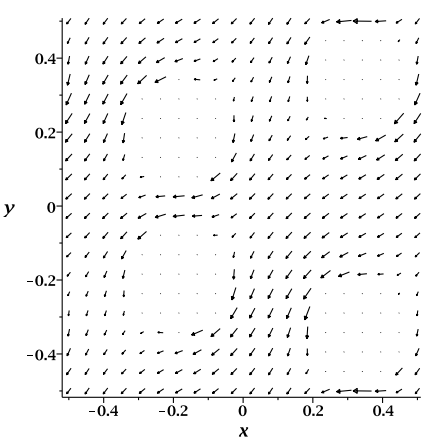

(b) $\theta=\pi / 4$

Figure 7. The flux distribution inside $Q_{(0,0)}$ for $\lambda_{k}=0.01, \gamma_{k}=100, \beta_{k}=-0.01$, $\theta=0 ; \pi / 4$.

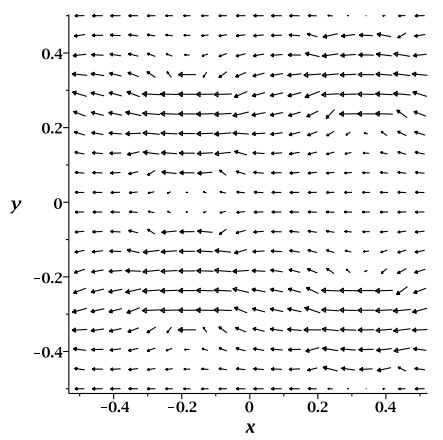

(a) $\theta=0$

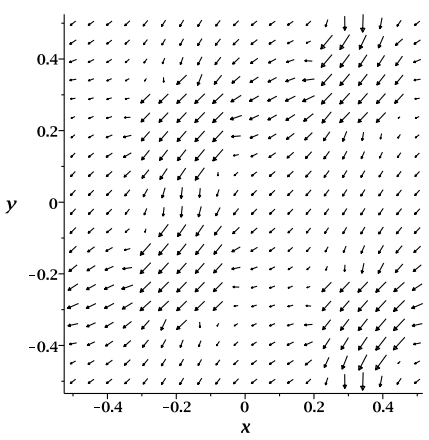

(b) $\theta=\pi / 4$

Figure 8. The flux distribution inside $Q_{(0,0)}$ for $\lambda_{k}=100, \gamma_{k}=100, \beta_{k}=-0.01$, $\theta=0 ; \pi / 4$.

Table 3. The components of the effective conductivity tensor $\Lambda_{e}$ for the configuration of the inclusions given in (4.1) for the material constants $\lambda_{k}=0.01, \gamma_{k}=100, \beta_{k}=-0.01$, $M=9$.

\begin{tabular}{lllll}
\hline$R$ & $\lambda_{e}^{x}$ & $\lambda_{e}^{x y}$ & $\lambda_{e}^{y x}$ & $\lambda_{e}^{y}$ \\
\hline 0.01 & 0.998768 & 0 & 0 & 0.998768 \\
0.05 & 0.949348 & $1.2 \cdot 10^{-7}$ & $1.2 \cdot 10^{-7}$ & 0.950053 \\
0.11 & 0.746523 & $3.4 \cdot 10^{-6}$ & $3.4 \cdot 10^{-6}$ & 0.765421 \\
0.135 & 0.624851 & $8.2 \cdot 10^{-6}$ & $8.2 \cdot 10^{-6}$ & 0.666135 \\
0.145 & 0.569849 & $1.2 \cdot 10^{-5}$ & $1.2 \cdot 10^{-5}$ & 0.624544 \\
\hline
\end{tabular}




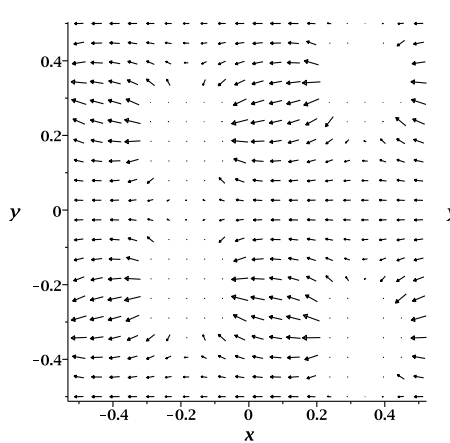

(a) $\theta=0$

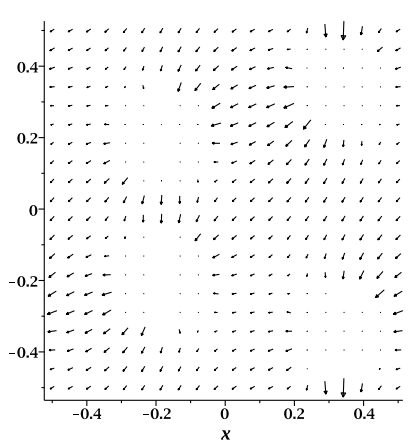

(b) $\theta=\pi / 4$

Figure 9. The flux distribution inside $Q_{(0,0)}$ for $\lambda_{k}=0.01, \gamma_{k}=100, \beta_{k}=-100$, $\theta=0 ; \pi / 4$.

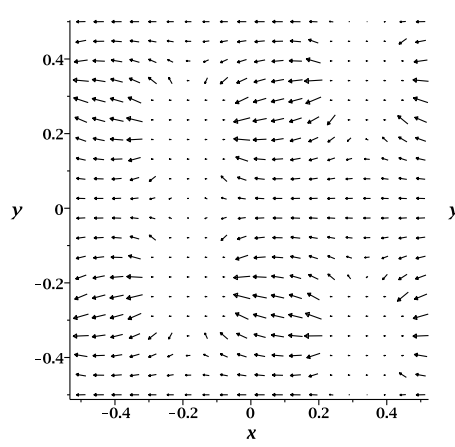

(a) $\theta=0$

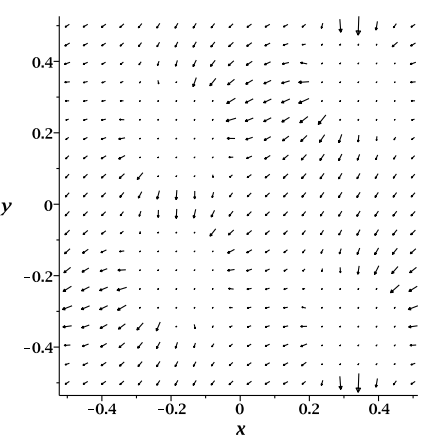

(b) $\theta=\pi / 4$

Figure 10. The flux distribution inside $Q_{(0,0)}$ for $\lambda_{k}=100, \gamma_{k}=100, \beta_{k}=-100$, $\theta=0 ; \pi / 4$.

Table 4. The components of the effective conductivity tensor $\Lambda_{e}$ for the configuration of the inclusions given in (4.1) for the material constants $\lambda_{k}=100, \gamma_{k}=100, \beta_{k}=-0.01$, $M=9$.

\begin{tabular}{lllll}
\hline$R$ & $\lambda_{e}^{x}$ & $\lambda_{e}^{x y}$ & $\lambda_{e}^{y x}$ & $\lambda_{e}^{y}$ \\
\hline 0.01 & 1.001233 & 0 & 0 & 1.001233 \\
0.05 & 1.052572 & $1.4 \cdot 10^{-7}$ & $1.4 \cdot 10^{-7}$ & 1.053354 \\
0.11 & 1.306471 & $6.0 \cdot 10^{-6}$ & $6.0 \cdot 10^{-6}$ & 1.339544 \\
0.135 & 1.501195 & $2.0 \cdot 10^{-5}$ & $2.0 \cdot 10^{-5}$ & 1.600389 \\
0.145 & 1.601164 & $3.3 \cdot 10^{-5}$ & $3.3 \cdot 10^{-5}$ & 1.754900 \\
\hline
\end{tabular}


Table 5. The components of the effective conductivity tensor $\Lambda_{e}$ for the configuration of the inclusions given in (4.1) for the material constants $\lambda_{k}=0.01, \gamma_{k}=100, \beta_{k}=-100$, $M=9$.

\begin{tabular}{lllll}
\hline$R$ & $\lambda_{e}^{x}$ & $\lambda_{e}^{x y}$ & $\lambda_{e}^{y x}$ & $\lambda_{e}^{y}$ \\
\hline 0.01 & 0.9999998 & $-3.8 \cdot 10^{-14}$ & $-3.8 \cdot 10^{-14}$ & 0.9999998 \\
0.05 & 0.999968 & $-1.3 \cdot 10^{-10}$ & $-1.3 \cdot 10^{-10}$ & 0.999968 \\
0.11 & 0.999631 & $-9.3 \cdot 10^{-9}$ & $-9.3 \cdot 10^{-9}$ & 0.999581 \\
0.135 & 0.999270 & $-3.8 \cdot 10^{-8}$ & $-3.8 \cdot 10^{-8}$ & 0.999089 \\
0.145 & 0.999063 & $-7.2 \cdot 10^{-8}$ & $-7.2 \cdot 10^{-8}$ & 0.998751 \\
\hline
\end{tabular}

Table 6. The components of the effective conductivity tensor $\Lambda_{e}$ for the configuration of the inclusions given in (4.1) for the material constants $\lambda_{k}=100, \gamma_{k}=100, \beta_{k}=-100$, $M=9$.

\begin{tabular}{lllll}
\hline$R$ & $\lambda_{e}^{x}$ & $\lambda_{e}^{x y}$ & $\lambda_{e}^{y x}$ & $\lambda_{e}^{y}$ \\
\hline 0.01 & 1.000024 & $3.6 \cdot 10^{-12}$ & $3.6 \cdot 10^{-12}$ & 1.000024 \\
0.05 & 1.002995 & $1.2 \cdot 10^{-8}$ & $1.2 \cdot 10^{-8}$ & 1.003062 \\
0.11 & 1.032965 & $8.1 \cdot 10^{-7}$ & $8.1 \cdot 10^{-7}$ & 1.037322 \\
0.135 & 1.063832 & $3.3 \cdot 10^{-6}$ & $3.3 \cdot 10^{-6}$ & 1.079309 \\
0.145 & 1.081262 & $6.0 \cdot 10^{-6}$ & $6.0 \cdot 10^{-6}$ & 1.107558 \\
\hline
\end{tabular}

\section{Conclusions}

As a result of our analysis the following conclusions can be formulated:

- We explicitly solved a complicated mixed boundary value problem for the Laplace equation with mixed soft and stiff imperfect contact conditions, serving as a model for a composite material structure.

- On the basis of this solution, we obtained in explicit form the material characteristics (such as temperature, flux and the effective conductivity) for a 2D composite material model with circular disjoint inclusions. The derived formulas contain all parameters of the considered model such as the conductivity, resistent coefficients, radii, centers of the inclusions, the conductivity of the matrix, and also special Eisenstein functions. It was illustrated that these formulas can be implemented in computational software.

- We also have shown the possibility to make the analysis of the model's properties by using their analytical representations.

\section{Acknowledgement}

This work was supported in part by the Portuguese Foundation for Science and Technology (FCT-Fundação para a Ciência e a Tecnologia) through the 
CIDMA - Centro de Investigação e Desenvolvimento em Matemática e Aplicações of University of Aveiro, Portugal, within project UID/MAT/04106/2013, as well as by the Shota Rustaveli National Science Foundation, with the grant number $31 / 39$.

\section{References}

[1] G. Allaire. Shape Optimization by the Homogenization Method. Springer Verlag, New York, 2002. http://dx.doi.org/10.1007/978-1-4684-9286-6.

[2] I.V. Andrianov, V. I. Bolshakov, V.V. Danishevskyy, and D. Weichert. Asymptotic study of imperfect interfaces in conduction through a granular composite material. Proceedings of the Royal Society A, 466(2121):2707-2725, 2010. http://dx.doi.org/10.1098/rspa.2010.0052.

[3] Y. Benveniste and T. Miloh. Imperfect soft and stiff interfaces in twodimensional elasticity. Mechanics of Materials, 33(6):309-323, 2001.

[4] L. Berlyand and V. Mityushev. Generalized Clausius-Mossotti formula for random composite with circular fibers. Journal of Statistical Physics, 102(1):115145, 2001. http://dx.doi.org/10.1023/A:1026512725967.

[5] L.P. Castro, D. Kapanadze and E. Pesetskaya. Effective conductivity of a composite material with stiff imperfect contact conditions. Mathematical Methods in the Applied Sciences, 38(18):4638-4649, 2015. http://dx.doi.org/10.1002/mma.3423.

[6] L.P. Castro, D. Kapanadze and E. Pesetskaya. A heat conduction problem of $2 \mathrm{D}$ unbounded composites with imperfect contact conditions. Zeitschrift fuer Angewandte Mathematik und Mechanik, 95(9):952-965, 2015. http://dx.doi.org/10.1002/zamm.201400067.

[7] L.P. Castro, E. Pesetskaya and S. Rogosin. Effective conductivity of a composite material with non-ideal contact conditions. Complex Variables and Elliptic Equations, 54(12):1085-1100, 2009. http://dx.doi.org/10.1080/17476930903275995.

[8] P. Drygas. A functional-differential equation in a class of analytic functions and its application. Aequationes Mathematicae, 73(3):222-232, 2007. http://dx.doi.org/10.1007/s00010-006-2865-3.

[9] P. Drygas. Functional-differential equations in Hardy-type classes. Trudy Instituta Matematiki, 15(1):105-110, 2007.

[10] P. Drygas and V. Mityushev. Effective conductivity of arrays of unidirectional cylinders with interfacial resistance. The Quarterly Journal of Mechanics and Applied Mathematics, 62(3):235-262, 2009. http://dx.doi.org/10.1093/qjmam/hbp010.

[11] R. Guinovart-Díaz, R. Rodríguez-Ramos, J. Bravo-Castillero, F.J. Sabina, R. Dario Santiago and R. Martínez Rosado. Asymptotic analysis of linear thermoelastic properties of fiber composites. Journal of Thermoplastic Composite Materials, 20(4):389-410, 2007. http://dx.doi.org/10.1177/0892705707079607.

[12] V.V. Jikov, S.M. Kozlov and O.A. Olejnik. Homogenization of Differential Operators and Integral Functionals. Springer-Verlag, Berlin, 1994. http://dx.doi.org/10.1007/978-3-642-84659-5. 
[13] D. Kapanadze, G. Mishuris and E. Pesetskaya. Improved algorithm for analytical solution of the heat conduction problem in composites. Complex Variables and Elliptic Equations, 60(1):1-23, 2015. http://dx.doi.org/10.1080/17476933.2013.876418.

[14] V. Mityushev. Transport properties of doubly periodic arrays of circular cylinders and optimal design problems. Applied Mathematics and Optimization, 44(1):1731, 2001. http://dx.doi.org/10.1007/s00245-001-0013-y.

[15] V. Mityushev. Exact solution of the R-linear problem for a disk in a class of doubly periodic functions. Journal of Applied Functional Analysis, 2:115-127, 2007.

[16] B. Noetinger. An explicit formula for computing the sensitivity of the effective conductivity of heterogeneous composite materials to local inclusion transport properties and geometry. Multiscale Modeling and Simulation, 11(3):907-924, 2013. http://dx.doi.org/10.1137/120884961.

[17] N. Rylko. Transport properties of a rectangular array of highly conducting cylinders. Journal of Engineering Mathematics, 38(1):1-12, 2000. http://dx.doi.org/10.1023/A:1004669705627.

[18] N. Rylko. Structure of the scalar field around unidirectional circular cylinders. Proceedings R. Soc. London A, 464(2090):391-407, 2008. http://dx.doi.org/10.1098/rspa.2007.0114.

[19] A. Weil. Elliptic Functions According to Eisenstein and Kronecker. Classics in Mathematics, Springer, Berlin, 1999. 